BÀN VỀ TIÊU CHUẨN ĐÁNH GIÁ CHẤT LỰ̛̣NG THÔNG TIN KẾ TOÁN

\title{
REVIEW OF EVALUATION CRITERIA FOR ACCOUNTING INFORMATION QUALITY
}

\author{
Phạm Quốc Thuần \\ Trường Đại học Kinh tế - Luật, ĐHQG - HCM - thuanpq@uel.edu.vn \\ (Bài nhận ngày 06 tháng 01 năm 2015, hoàn chỉnh sửa chũa ngày 02 tháng 03 năm 2015)
}

\section{TÓM TẮT}

Nghiên cứu về chất luợng thông tin (CLTT) là một trong nhũng vấn đề được quan tâm nhiều nhất trong lĩnh vục hệ thống thông tin kế toán (AIS - Accounting Information System) (Foong, 1999). Trong nghiên cứu về CLTT, nghiên cứu về đo luờng CLTT khá đa dạng, cả về số luợng lẫn quan điểm. Thông qua việc tổng hợp các công trình nghiên cứu về CLTT tù truớc đến nay, chúng tôi tiến hành xác định xu huớng phát triển của các nghiên cứu về đo luờng CLTT tù̀ đó xác định mô hình đo luờng CLTT phù hợp với nghiên cứu trong lĩnh vục kế toán.

Tù khóa: Thông tin, chất luợng thông tin, hệ thống thông tin, hệ thống thông tin kế toán, hệ thống thông tin quản lý, kế toán tài chính.

\section{ABSTRACT}

Information quality has been the focus of research in the field of accounting information system (AIS) (Foong, 1999). Studies on information quality are diverse in both quantities and viewpoints. Through a review of extant studies on information quality, we aim to define the trend of studies on information quality, thereby building a suitable evaluation model of information quality in the field of accounting.

Key words: Information, information quality, information system, accounting information system, information management system, financial accounting.

\section{CÁC KHÁI NIỆM}

\subsection{Thông tin}

Khái niệm về thông tin hết sức đa dạng. Chaker Haouet (2008) cho rằng không tồn tại một khái niệm duy nhất cho thông tin, các khái niệm thay đổi theo lĩnh vực được tham chiếu. Trong lĩnh vực MIS, Zmud (1978) cho rằng thông tin được thể hiện ở dạng các báo cáo được in ở các định dạng khác nhau; English
(1999) cho rằng thông tin được thể hiện ở dạng nội dung cơ sở dữ liệu và kho dữ liệu hoặc đầu ra,,..

Trong lĩnh vực AIS, thông tin kế toán được xác định rõ trong các khái niệm về kế toán. Một cách tổng quát, thông tin kế toán bao hàm những thông tin kinh tế, tài chính dưới hình thức giá trị, hiện vật và thời gian lao động (Luật Kế toán Việt Nam, 2003). 
Trong bài viết này, chúng tôi khái niệm thông tin trong lĩnh vực AIS là dựa trên quan điểm của Luật Kế toán Việt Nam.

\subsection{Chất lượng}

Cũng như khái niệm về thông tin, có rất nhiều khái niệm về chất lượng với ít sự đồng thuận (Crosby, 1995). Tùy thuộc quan điểm về lĩnh vực nghiên cứu mà khái niệm về chất lượng cũng thay đổi. Trong lĩnh vực kế toán, chất lượng được khái niệm gắn với nhu cầu của người sử dụng thông tin. Theo quan điểm hòa hợp giữa IASB và $\mathrm{FASB}$ thì chất lượng của kế toán được thể hiện ở mục tiêu cung cấp thông tin tài chính hữu ích cho những nhà đầu tư, người cho vay, các chủ nợ khác hiện hữu và tiềm năng trong việc ra quyết định về việc cung cấp nguồn lực cho một thực thể (IASB, 2010).

Trong nghiên cứu này, chúng tôi khái niệm chất lượng trong lĩnh vực kế toán dựa trên quan điểm của $\mathrm{IASB}$ và $\mathrm{FASB}$.

\subsection{Chất lượng thông tin}

Về khía cạnh thông tin, CLTT được định nghĩa như là thông tin đáp ứng những đặc điểm kỹ thuật hay yêu cầu (Kahn và Strong, 1988). Về khía cạnh người sử dụng, CLTT được định nghĩa như là thông tin phù hợp đối với người sử dụng thông tin (Wang vàStrong, 1996). Trong nghiên cứu của mình, chúng tôi đứng trên quan điểm người sử dụng để khái niệm CLTT.

CLTT trong lĩnh vực kế toán được xác định rõ trong khuôn mẫu các khái niệm của kế toán (Conceptual Framework). Theo Luật Kế toán Việt Nam, các thuộc tính tác động đến chất lượng thông tin kế toán bao gồm: trung thực, khách quan, đầy đủ, kịp thời, dễ hiểu, có thể so sánh (Luật Kế toán Việt Nam, 2003); Theo quan điểm hòa hợp giữa $\mathrm{IASB}$ và $\mathrm{FASB}$ cho rằng nếu thông tin tài chính là hữu ích, nó cần phải thích đáng và được thể hiện trung thực, ...
Trong phạm vi bài viết, chúng tôi khái niệm CLTT dựa trên quan điểm của IASB và $\mathrm{FASB}$, theo đó, chất lượng thông tin được đo lường bằng thích đáng và trình bày đúng sự thật của các thông tin trên báo cáo tài chính.

\section{CÁC MÔ HÌNH ĐO LƯờng CHẤT LƯợNG THÔNG TIN}

\subsection{Các hướng nghiên cứu về chất lượng thông tin}

Nghiên cứu về CLTT đã được đề cập từ những năm 60 và được tiến hành một cách rộng rãi trong vòng hai thập kỷ gần đây. Nghiên cứu về CLTT được phân thành hai lĩnh vực chủ yếu: lĩnh vực MIS và AIS. Dựa vào đặc điểm trên chúng tôi tiến hành xem xét các lý thuyết xuất phát chủ yếu từ các lĩnh vực MIS và AIS và đây cũng là căn cứ cho phép chúng tôi xác định mô hình đo lường CLTT thông qua cái nhìn đa dạng của liên ngành với trọng tâm là MIS và AIS.

\subsection{Mô hình đo lường CLTT trong lĩnh} vực MIS

2.2.1. Tổng quan về nghiên cúu CLTT trong lĩnh vục MIS

Vào những năm 60 , khi mà MIS bắt đầu được sử dụng nhiều hơn và được xem như là một công cụ quản lý, chất lượng của hệ thống liên quan đến nhu cầu và khả năng quản lý là một vấn đề được quan tâm (Ackoff, 1967). Sự quan tâm đến việc đánh giá MIS ngày càng phát triển mạnh mẽ (Chervany,1970) và được tiếp cận bằng cách đo lường hiệu quả dưới nhiều cách thức khác nhau của MIS, chẳng hạn như đánh giá hệ thống, đánh giá giá trị sử dụng của hệ thống (Gallagher, 1974), đánh giá sự hài lòng của người sử dụng đối với hệ thống (Bailey và Pearson, 1983).

Trong nghiên cứu của mình, với mục tiêu xây dựng một khuôn mẫu cho CLTT, chúng tôi đã tiến hành liệt kê tất cả các nghiên cứu về MIS kèm theo minh họa chi tiết các thành phần 
CLTT. Kết quả thống kê cho thấy trong khoảng thời gian bắt đầu từ những năm 60 (là thời điểm ghi nhận sự phát triển trong nghiên cứu về MIS) cho đến nay, các nhà nghiên cứu về CLTT trong lĩnh vực MIS đã đưa ra đến 67 tiêu chí để đo lường CLTT. Tuy nhiên, trong số nghiên cứu về CLTT đã được liệt kê, mô hình nghiên cứu của Wang và cộng sự (1995) và được thực nghiệm cụ thể hóa bởi Wang và Strong (1996) được xem là thống trị về khía cạnh lý thuyết CLTT trong lĩnh vực MIS. Các mô hình lý thuyết này chứa đựng rất nhiều các thuộc tính quan trọng và có điểm giao thoa nhiều nhất với mô hình CLTT đứng trên quan điểm được công bố của IASB \& FASB 2010.

\subsubsection{Mô hình đo lưòng CLTT trong lĩnh} vuc MIS

Mô hình đo lường CLTT của Wang và cộng sự (1995)

Wang và cộng sự (1995) đã trình bày một bản tóm tắt các mô hình CLTT có thứ bậc của họ. Trong mô hình này, CLTT có cấu tạo bao hàm bốn thành phần chính: Khả năng truy cập dữ liệu phải có khả năng truy cập; có thể diễn giải được - cú pháp và nội dung phải dễ hiểu; hữu dụng - ghi nhận kịp thời các biến động và đáng tin cậy (được đo lường bởi "đầy đử, "nhất quán", "có nguồn gốc" và "chính xác") Xem Sơ đồ 1 .

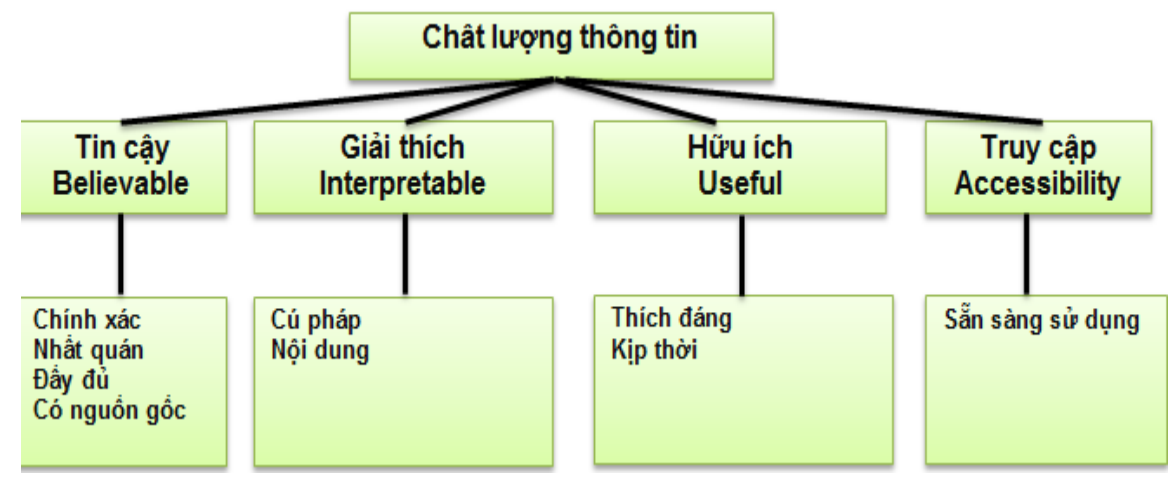

So đồ 1. Mô hình về khuôn mẫu CLTT (Wang và cộng sự 1995)

Mô hình đo lường CLTT của Wang và Strong (1996)

Wang và Strong (1996) đã trình bày kết quả của nghiên cứu khảo sát trong đó một loạt các thuộc tính được dùng để xác định CLTT được đánh giá bởi người sử dụng. Kết quả nghiên cứu cho thấy CLTT được cấu tạo bởi 12 thành phần - Xem sơ đồ 2 .

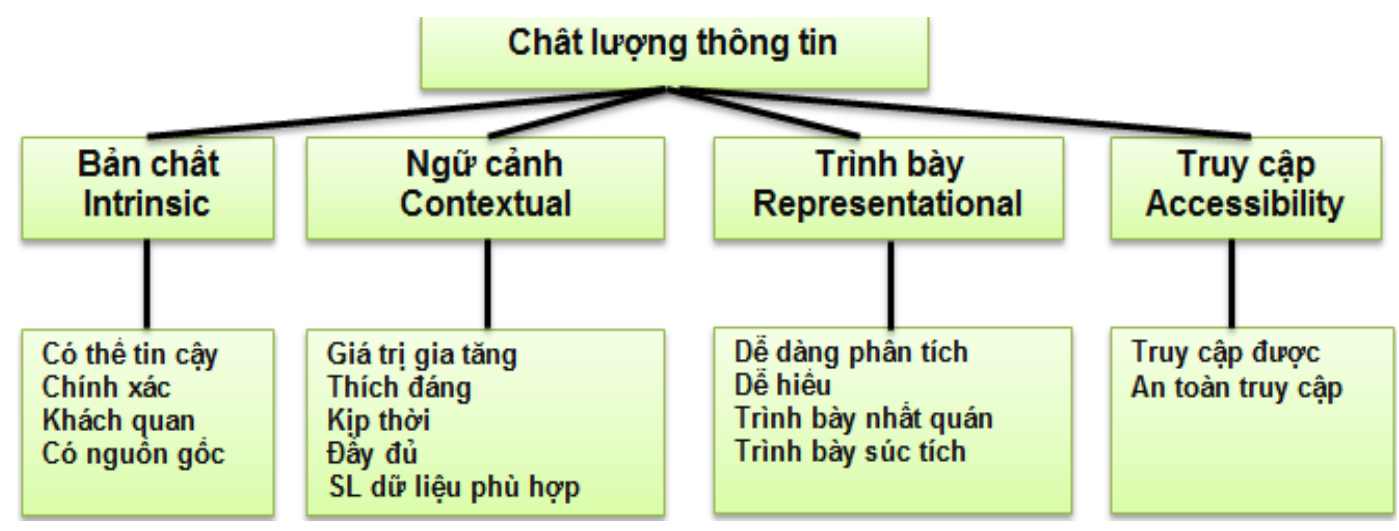

Sơ đồ 2. Mô hình về khuôn mẫu CLTT (Wang và Strong 1996)

\section{Trang 70}




\subsection{Mô hình đo lường CLTT trong lĩnh} vục AIS

2.3.1. Tổng quan về nghiên cưu CLTT trong lĩnh vục AIS

Vào năm 1966, Hiệp hội Kế toán Mỹ (AAA) đã công bố một báo cáo về nền tảng lý thuyết của kế toán (A statement of Basic Accounting Theory) (Paul E. Fertig, 1967) và nêu rõ rằng mục tiêu chính của lý thuyết kế toán là phải đưa ra được các thuộc tính của thông tin nhằm đảm bảo tính hữu ích đối với người sử dụng thông tin. Công bố của AAA (1966) đã được ca ngợi như là một cột mốc quan trọng đánh dấu việc hướng tới một hệ thống lý thuyết của kế toán được xem là "xác định, đo lường và trao đổi thông tin kinh tế hỗ trợ cho việc ra quyết định của người sử dụng thông tin" (Sterling, 1967).

Dựa trên nền tảng lý thuyết về tính hữu ích của thông tin được cung cấp bởi AAA (1966, 1972), FASB (1993, 2010) đã có rất nhiều nghiên cứu hướng đến việc làm rõ các thành phần và tiêu chuẩn cấu thành nên CLTT kế toán và các thành phần, tiêu chuẩn được các nhà nghiên cứu đưa ra rất đa dạng. Tuy nhiên các thành phần CLTT theo mô hình của FASB,
IASB được các nhà nghiên cứu hướng đến nhiều nhất.

\subsubsection{Các mô hình nghiên cưu tiêu biểu} về CLTT trong lĩnh vưc AIS

\section{Mô hình của FASB}

Mô hình của FASB thiết lập các thuộc tính hữu ích của thông tin được ghi nhận trong các tài liệu kế toán thành một hệ thống khái niệm hướng tới cung cấp thông tin cho việc ra quyết định kinh tế. Người sử dụng thông tin được coi là người đưa ra quyết định tổng thể về lợi ích của bất kỳ thông tin mà họ nhận được. Thông tin phải liên quan đến lĩnh vực và mục đích của người sử dụng cũng như cung cấp những tiên đoán và phản hồi của người sử dụng và luôn luôn sẵn sàng được cung cấp cho người sử dụng thì được xem là thích đáng. Thông tin trình bày một cách trung thực, trung lập nội dung của sự kiện và có đầy đủ bằng chứng để kiểm tra tính xác thực thì được xem là đáng tin cậy (Reliable). Tính thích đáng và tính đáng tin cậy có mối quan hệ với sự nhất quán của nó trong việc đo lường, trình bày và so sánh các kết quả của một doanh nghiệp theo thời gian và giữa các doanh nghiệp với nhau. Mô hình của FASB được trình bày ở sơ đồ 3 .

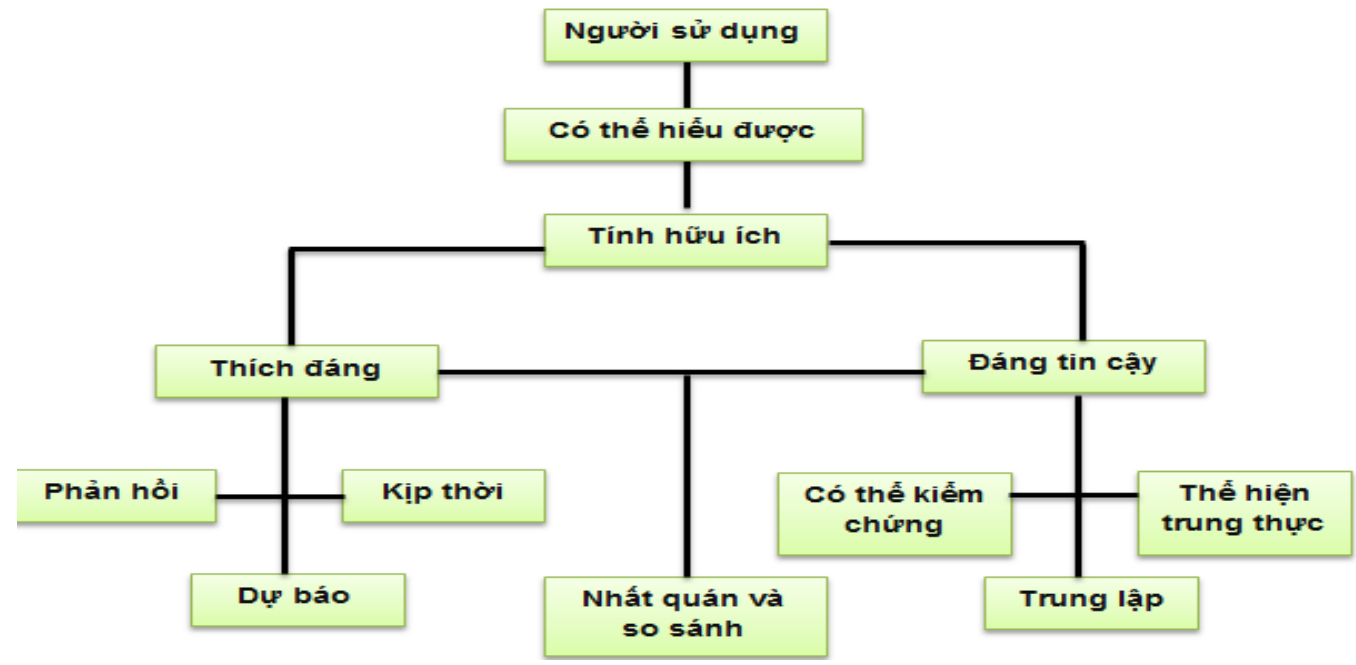

Sơ đồ 3. Mô hình CLTT của FASB (1980, 1993) 


\section{Mô hình của IASB}

Vào tháng 4 năm 2001, IASB đã đưa ra một khuôn khổ các khái niệm làm nền tảng cho việc lập và trình bày báo cáo tài chính. Liên quan đến các thuộc tính thuộc CLTT kế toán, khuôn mẫu khái niệm của $\mathrm{IASB}$ cho rằng chất lượng được hiểu như là những thuộc tính làm cho những thông tin trình bày trên các báo cáo tài chính trở nên hữu ích đối với những người sử dụng thông tin. IASB đã đưa ra bốn đặc trưng chính có liên quan đến chất lượng của thông tin kế toán: Tính dễ hiểu; tính thích đáng; tính đáng tin cậy; tính có thể so sánh (xem mô hình ở sơ đồ 4).

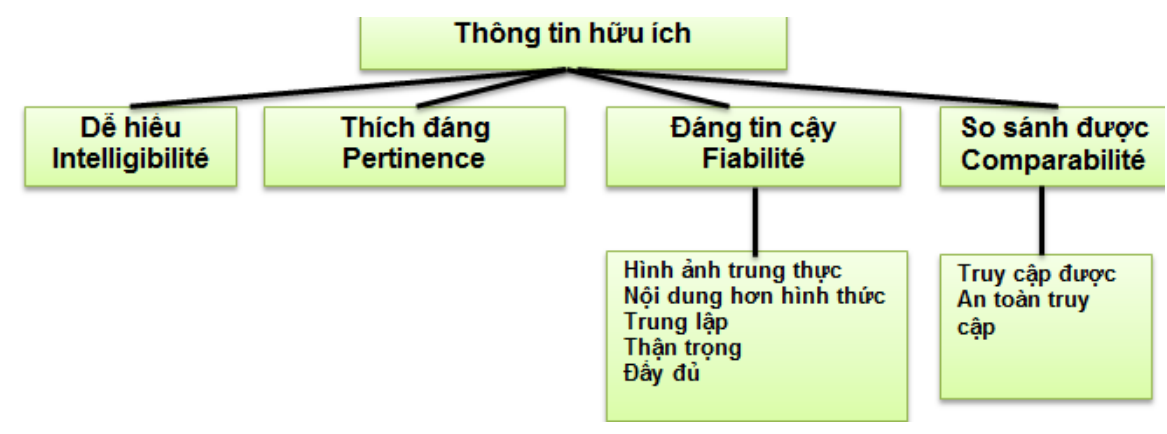

Sơ đồ 4. Mô hình CLTT của IASB (2001)

\section{Mô hình hài hòa giữa FASB và IASB}

Thỏa thuận Norwalk được ký ngày 18 tháng 11 năm 2002 giữa FASB và IASB đã đặt nền móng của dự án hội tụ giữa hai cơ quan thiết lập chuẩn mực kế toán lớn nhất thế giới. Vào tháng 10 năm 2004, hai tổ chức này đã thành lập một đề án nhằm thiết lập những tiêu chuẩn cho việc trình bày thông tin trên $\mathrm{BCTC}$ nhằm hướng đến việc cải thiện tính hữu ích của thông tin trên BCTC cũng như phát triển một khuôn khổ khái niệm chung giữa hai tổ chức. Vào cuối tháng 10/2010, Ủy ban đã hoàn tất giai đoạn $\mathrm{A}$ của dự án, đó là thống nhất vấn đề về mục tiêu và các thuộc tính liên quan đến CLTT hữu ích. Các thuộc tính chất lượng của thông tin hữu ích theo quan điểm của IASB\& FASB 2010 được trình bày tại chương 3 của báo cáo. Báo cáo đã khẳng định rằng tính thích đáng và thể hiện trung thực là đặc tính chất lượng nền tảng của thông tin kế toán, cụ thể:

- Thích đáng (relevance): thích đáng của thông tin được xem là khả năng tác động của thông tin đến việc ra quyết định của người sử dụng. Thông tin có thể có khả năng ảnh hưởng đến việc ra quyết định khi nó mang giá trị dự đoán (predictive value), giá trị xác nhận (confirmatory value), hay cả hai.

- Thể hiện trung thục (faithful representation): BCTC trình bày các hiện tượng kinh tế dưới dạng từ ngữ và các con số. Để đảm bảo hữu ích, thông tin tài chính không chỉ trình bày các hiện tượng một cách thích đáng mà còn phải trình bày đúng sự thật các hiện tượng cần được trình bày. Để đạt được điều đó, thông tin cần phải mang tính toàn vẹn (complete), trung lập (neutral) và không sai sót (free from error).

Các thuộc tính làm gia tăng CLTT: Có khả năng so sánh (comparability), có thể kiểm chứng (verifiability), kịp thời (timeliness) và dễ hiểu (understandability) là những thuộc tính chất lượng làm gia tăng sự hữu ích của thông tin.

Các đặc điểm CLTT theo quan điểm dự án hội tụ giữa IASB và FASB theo Sơ đồ 5

\section{Trang 72}




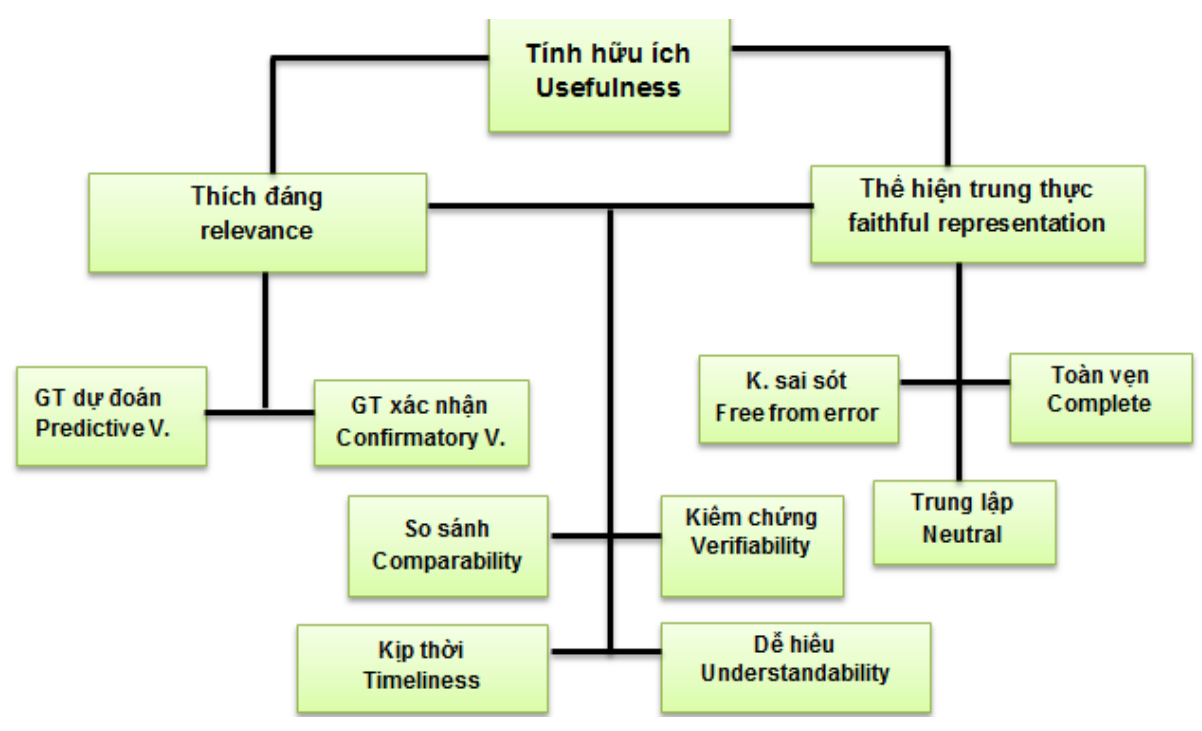

Sơ đồ 5. Mô hình CLTT kế toán của IASB \& FASB 2010

\section{XÁC ĐỊNH MÔ HÌNH ĐO LƯờng CLTT TRONG LĨNH VỰC KẾ TOÁN}

Nghiên cứu về CLTT từ lúc bắt đầu (vào những năm 60) đến nay đã và đang là một đề tài được nhiều nhà nghiên cứu quan tâm. Bên cạnh đó, trong các công bố của các tổ chức nghề nghiệp kế toán; các văn bản pháp lý về kế toán của các cơ quan Nhà nước cũng đưa ra các khuôn mẫu về CLTT.

Xuất phát từ đặc điểm khác biệt, sự phân nhánh trong nghiên cứu về MIS và AIS cũng như đúc kết từ các kết quả nghiên cứu về CLTT trước đó, chúng tôi kết luận rằng có sự tồn tại khác biệt về mô hình đo lường CLTT trong lĩnh vực MIS và AIS. Vì vậy, trong nghiên cứu về CLTT, cần phải xác định phạm vi nghiên cứu, lĩnh vực nghiên cứu cụ thể để vận dụng mô hình đo lường CLTT phù hợp. Đối với nghiên cứu CLTT trong lĩnh vực kế toán, mô hình đo lường CLTT theo quan điểm hòa hợp giữa IASB và FASB là mô hình phù hợp nhất vì các lý do sau:

- Mô hình đo lường về CLTT được công bố bởi IASB (2001) và FASB (1980, 1993) là mô hình đạt được sự đồng thuận của phần lớn các nhà nghiên cứu trong lĩnh vực AIS.

- Kế toán là lĩnh vực chịu sự chi phối cao về các chuẩn mực, nguyên tắc được ban hành bởi các cơ quan Nhà nước và các tổ chức nghề nghiệp, vì vậy mô hình về CLTT dựa trên quan điểm hội tụ của IASB \& FASB được công bố bởi IASB vào năm 2010 được phần lớn các tổ chức Nhà nước và các tổ chức nghề nghiệp trong lĩnh vực kế toán chấp thuận và tham chiếu khi đề ra khuôn mẫu khái niệm về CLTT.

- Quan điểm hòa hợp giữa IASB và FASB phù hợp với xu hướng hòa hợp và hội tụ quốc tế về chuẩn mực kế toán.

\section{KẾT LUẬn}

Mặc dù nghiên cứu về CLTT đã có một quá trình phát triển khá lâu dài trên Thế giới - xét trong lĩnh vực MIS lẫn AIS, tuy nhiên vẫn còn chưa được các nhà nghiên cứu trong lĩnh vực kế toán Việt Nam quan tâm đúng mức. Bài viết của chúng tôi đã đúc kết và tìm ra xu hướng phát triển của nghiên cứu về CLTT, làm rõ 
những điểm tương đồng và khác biệt đối với nghiên cứu về CLTT trong hai lĩnh vực MIS và AIS, từ đó đã xác định mô hình đo lường
CLTT theo quan điểm hòa hợp giữa IASB \& FASB là mô hình phù hợp cho việc đo lường CLTT kế toán.

\section{TÀI LIỆU THAM KHẢO}

[1]. Ackoff, RL. (1967), Management Misinformation Systems, Management Science 14 (4): B147-B156.

[2]. Baily, J.E. and S.W. Pearson (1983), Development of a Tool For Measuring and AnalyzingComputer User Satisfaction, Management Science 29(5): 530-545.

[3]. Chaker Haouet (2008), Informatique décisionnelle et management de la performance de l'entreprise, Laboratoire Orléanais de gestion IAE d'Orléans.

[4]. Chervany, N. L. and G. W. Dickson (1970), Economic Evaluation of Management Information Systems: An Analytical Framework, Decision Sciences, 1. 296-308.

[5]. Crosby, Philip B. (1995), Quality Is Still Free; Making Qualitv Certain in Uncertain Times, McGraw-Hill, New York, USA.

[6]. English, L. p. (1999),Improving Data Warehouse and Business Inionnation Quality: Methodes for Reducing Costs and Increasing Profits, John Wiley and Sons, Inc, New York, New York, USA.

[7]. Foong, S. Y. 1999, Effect of end-user personal and systems attributes on computer-based information systemsuccess in Malaysian SMEs, Journal of Small Business Management. July: 81-87.
[8]. Gallagher, C.A. (1974), Perceptions of the Value o $f$ a Management Information System, The Academy of Management Journal 17(1):46-55.

[9]. IASB (2010), Conteptual Framework for Financial Reporting 2010, IFRS Foundation chapter 1, OB 2; chapter 3, QC 4.

[10]. Kahn, B.K. and Strong, D.M. (1998), Product and service performance model for information quality: an update.4th International Conference on Information Quality,Boston, Massachusetts, USA.

[11]. Paul E. Fertig (1967), A Satement of Basic Accounting Theory, Proquest Central sep 1967, p 663- 671.

[12]. Proquest central (2011), IASB Conceptual framework, Charter 2011 p 70.

[13]. Quốc Hội nước CHXHCN Việt Nam (2003), Luật Kế toán.

[14]. Wang, R.Y., Storey, V. c. and c. p. Firth, c.p. (1995), A Framework for Analysis of DataQuality Research, IEEE Transactions on Knowledge and Data Engineering 7(4):623- 640.

[15]. Wang, R.Y. and D.M. Strong (1996), Beyond Accuracy, What Data Quality Means to Data Consumers,Journal of Management Information Systems 12(4):5-34. 